\title{
Analyticity and Probability Properties of One-Dimensional Brownian Motion
}

\author{
Abolghassem Ghaffari
}

(July 11, 1961)

\begin{abstract}
The most general solutions of one-dimensional Brownian motion, governed by the Chapman-Kolmogoroff functional equation, in the form of series of products of two Laguerre polynomials are described. Some analytical and probabilistic properties of these solutions are discussed and it is shown that these solutions are different from those given by A. N. Kolmogoroff. The limiting behavior of the probability function is investigated. A derivation of the corresponding parabolic partial differential equation is obtained.
\end{abstract}

\section{Introduction}

The mathematical study of Brownian motion and diffusion phenomena in the case of a nonuniform fluid led S. Chapman $[1]^{1}$ to introduce the functional equation

$$
f\left(\rho, \tau+\tau_{0}, r, t\right)=\int f\left(\rho^{\prime}, \tau, r, t\right) f\left(\rho-\rho^{\prime}, \tau_{0}, r+\rho^{\prime}, t+\tau\right) d \rho^{\prime} .
$$

B. Hostinsky [11] in studying the functional equation of M. Smoluchowski [19] for the same problem, observed that the functional equation of Chapman was a generalization of the functional equation of Smoluchowski, and found the general solution of the Chapman equation by using the method of integration by substitutions due to V. Volterra.

The same author [11] puts the functional equation of Chapman in the more symmetric form

$$
f\left(x_{1}, x_{3}, t_{1}, t_{3}\right)=\int_{a}^{b} f\left(x_{1}, x_{2}, t_{1}, t_{2}\right) f\left(x_{2}, x_{3}, t_{2}, t_{3}\right) d x_{2}
$$

which is equivalent to that of Chapman for the case where the space in which the Brownian motion or diffusion phenomena take place has only one dimension.

On the other hand, A. N. Kolmogoroff [17] has considered directly a very general functional equation valid for a domain of any number of dimensions, which reduces to eq (2) in the case of a single dimension and which can be considered as a very general form of one of the conditions which occurs in the problem of chain probabilities studied by Markoff.

Moreover, A. Kolmogoroff has given particular solutions of eq (2) and has shown that very wide classes of his solutions satisfy linear partial differential equations of the second order and parabolic type, and generalized the functional equation of Chapman from the special problems of Brownian motion and diffusion phenomena to chain probabilities. The eq (2) is commonly called the Chapman-Kolmogoroff functional equation.

Nevertheless, the most general solution of the Chapman-Kolmogoroff equation has not been given. M. Fréchet, however, has given very general solutions having forms different from the forms given by the preceding authors.

M. Fréchet has found two methods: the first [6] is based on a procedure used in mathematical physics and the second [7] on reducing the study of the most general solutions which are doubly square integrable to the corresponding study in the discontinuous case. The two methods provide two distinct forms whose equivalence is neither obvious nor certain.

The most general solutions of the Chapman-Kolmogoroff equation in one-dimensional space have been given by M. Fréchet and the present author. In fact, the case of the finite interval $(0,2 \pi)$ has been treated by M. Fréchet [6], and the case of a region infinite in both directions has been investigated in detail $[8,10]$.

1 Figures in brackets indicate the literature references at the end of this paper. 
In this paper some analytical and probabilistic properties of the most general solutions of one-dimensional Brownian motion in the interval $(0, \infty)$ are described. The corresponding parabolic partial differential equation, which constitutes the fundamental equation of Brownian motion and diffusion theory, is obtained, and the limiting behavior of the solutions at infinity is analyzed.

\section{Statement of the Problem and Formulation of the Fundamental Results}

Let us consider a free spherical Brownian particle (i.e., in the absence of an external field force) depending on a finite number $\nu$ of parameters and which can assume all states $Q$, forming a certain set $V$ (which one can consider as a region of $\nu$-dimensional Euclidean space). One assumes then that there exists a probability $\omega(M, s ; \nu, t)$ that the Brownian particle passes from state $M$ at the instant $s$ to any one of the states of a set $\nu$ at the later instant $t$.

The probability function $\omega(M, s ; \nu, t)$ is, by virtue of the total probability theorem, an additive function of the set $\nu$. It is clear that

where

$$
\omega(M, s ; \nu, s)=\delta(M, \nu)
$$

$$
\delta= \begin{cases}1 & \text { if } M \epsilon \nu \\ 0 & \text { if } M \notin \nu\end{cases}
$$

When $s<t$, one can consider the simple case where the additive function of the set $\nu$ can be represented by a Lebesgue integral

$$
\omega(M, s ; \nu, t)=\int_{\nu} f(M, s ; Q, t) d Q
$$

where $d Q$ is an element of the $\nu$-dimensional space and $\int_{\nu}$ is a $\nu$-tuple integral.

When this assumption is made, one finds, in applying total and compound probability theorems that the Brownian displacement function $f$, which govern the $\nu$-dimensional Brownian motion, ${ }^{2}$ satisfies the Chapman-Kolmogoroff functional equation

$$
f(M, s ; P, t)=\int_{V} f(M, s ; Q, u) f(Q, u ; P, t) d Q
$$

for $s<u<t . \quad V$ denotes a $\nu$-dimensional Euclidean space and $f$ is Lebesgue-measurable in $V$.

In the problem of chain probabilities, $f(M, s ; P, t) d P$ is the elementary probability that the state of a physical system, fixed by $\nu$ parameters, and represented by $M$ at the instant $s$, becomes at the later instant $t$ one of the states of a certain elementary set $d P$ of states. However, in this interpretation the transition probability or density probability function $f(M, s ; P, t)$ must satisfy the probabilistic conditions

$$
\begin{gathered}
f(M, s ; P, t) \geq 0, \\
\int_{V} f(M, s ; P, t) d P=1 .
\end{gathered}
$$

If the physical system is moving continuously it has to travel the finite distance $M P$ during a finite time $t$ different from $s$. Then if the states $M$ and $P$ are two different states we have

$$
\lim _{t \rightarrow s} f(M, s ; P, t)=0, \quad M \neq P .
$$

If the state $P$ coincides with the state $M$, the transition probability $f$ is no more continuous and it tends to infinity as $t \rightarrow s$

$$
\lim _{P \rightarrow M, t \rightarrow s} f(M, s ; P, t)=\infty .
$$

2 By a $\nu$-dimensional Brownian motion we understand a $\nu$-dimensional strong Markoff process whose proiections are all one-dimensional Brownian motion. 
Such properties of the transition probability function $f$, when $t \rightarrow s$, resemble those of the Dirac $\delta$-function which is a kind of improper function. It follows that the condition (8) must be excluded from chain probabilities. Therefore the assumption of the existence of an elementary probability $f(M, s ; P, t) d P$ cannot be accepted completely and an exception must be made for the case $s=t, M=P$. If $f$ is interpreted as a generalized function, in the sense of the distribution theory, then the case $s=t$ and $M=P$ is covered by the statement that $f$ has $\delta$-function behavior in this case.

Using the additive theory, both N. Wiener [23] and P. Lévy [18] obtained very important results concerning the transition probability function. M. Kac [12], S. Kakutani [14, 15] and others in a series of papers obtained properties of the Brownian motion in a $\nu$-dimensional space and in connection with the Riemann surfaces. An interesting probabilistic discussion of the general diffusion process in one dimension is also given by W. Feller [5]. Recently M. Kac [13], relying on the properties of Wiener measure in the space of continuous functions, showed the disadvantage of Wiener measure theory applied to Brownian motion.

On the purely physical side the two papers by Uhlenbeck and Ornstein [21] and Uhlenbeck and M. C. Wang [22] should be mentioned.

\section{Method of Solution}

To obtain a very general solution of the functional eq (4), M. Fréchet [6] tried a method of separation of variables in the form

$$
f(M, s ; P, t)=\sum_{i=1}^{n} A_{i}(M, s) B_{i}(P, t)
$$

and found that the necessary and sufficient condition that the expression (9) should be a solution of eq (4) is that, for the values of $u$ such that $s<u<t$, the functions $A_{i}(Q, u)$ and $B_{i}(Q, u)$ form a biorthonormal system over $V$, i.e.,

$$
\int_{V} A_{i}(Q, u) B_{j}(Q, u) d Q=\delta_{i j} \text { where } \delta_{i j}= \begin{cases}1 & \text { if } i=j \\ 0 & \text { otherwise. }\end{cases}
$$

Nevertheless, Fréchet realized that the expression (9) does not satisfy the conditions of chain probabilities and introduced instead the infinite series

$$
f(M, s ; P, t)=\sum_{n=0}^{\infty} A_{n}(M, s) B_{n}(P, t)
$$

and proved that the necessary and sufficient condition that the transition probability function $f(M, s ; P, t)$, satisfying the Chapman-Kolmogoroff eq (4), could be written, in at least one way, in the form (11) where the convergence is uniform for $s<t$, is that the functions $A_{i}(M, s)$, $B_{i}(P, t)$ form a complete orthonormal set over $V$.

The series solution (11) is supposed to be uniformly convergent for $s$ and $t$ fixed such that $s<t$, and $M, P$ varying arbitrarily over $V$.

\section{One-Dimensional Case}

Considering the case of one-dimensional space $(\nu=1)$, and with the usual notations $x$, $y$, and $z$ for $M, P$, and $Q$, the Chapman-Kolmogoroff eq (4) can be written in the form

$$
f(x, s ; y, t)=\int_{V} f(x, s ; z, u) f(z, u ; y, t) d z, \quad s<u<t .
$$

Fréchet's method led us to take for the solution of eq (12) the infinite series

$$
f(x, s ; y, t)=\sum_{n=0}^{\infty} A_{n}(x, s) B_{n}(y, t)
$$


where the functions $A_{n}, B_{n}$ form a biorthonormal set over $V$.

The case of the finite interval $V(0,2 \pi)$ has been treated by M. Fréchet [6]. The solution of eq $(12)$ on $(-\infty, \infty)$ in the form of a series of products of two Hermite polynomials has been given by the author $[8,10]$, and its analytical and probabilistic properties are discussed in detail.

The most general solution of eq (12) in the interval $(0, \infty)$ is also given by the author $[8,9]$ in the form

$f(x, s ; y, t)=\sum_{n=0}^{\infty} \psi_{n}^{(\alpha)}(x) \psi_{n}^{(\alpha)}(y) \theta^{n}(s, t)=\exp \left(-\frac{x+y}{2}\right)(x y)^{\frac{\alpha}{2}} \sum_{n=0}^{\infty} \lambda_{n}^{-1} L_{n}^{(\alpha)}(x) L_{n}^{(\alpha)}(y) \theta^{n}(s, t), \alpha>-1$,

where the infinite sequence of $L^{2}$-functions $\left\{\psi_{n}^{(\alpha)}(x)\right\}$

$$
\psi_{n}^{(\alpha)}(x)=\lambda_{n}^{-\frac{1}{2}} \exp \left[-\frac{x}{2}\right] x^{\frac{\alpha}{2}} L_{n}^{(\alpha)}(x)
$$

form a complete orthonormal set over $(0, \infty)$, and $\underset{n}{L_{n}^{(\alpha)}}(x)$ is the $n$th associated Laguerre polynomial defined by

In addition,

$$
L_{n}^{(\alpha)}(x)=\frac{x^{-\alpha} e^{x}}{n !} \frac{d^{n}}{d x^{n}}\left(x^{n+\alpha} e^{-x}\right) .
$$

$$
\theta(s, t)=\frac{a(s)}{a(t)}<1, \quad \text { for } s<t,
$$

$a(s)$ is a positive increasing continuous function $\neq 0$, chosen arbitrarily, and

$$
\lambda_{n}=\frac{\Gamma(n+\alpha+1)}{\Gamma(n+1)},
$$

where $\Gamma$ denotes the gamma function.

It has been shown $[8,9]$ that, for $s, t$ fixed such that $s<t$ and $x, y$ varying arbitrarily over $(0, \infty)$, the series solution (13) is absolutely convergent in $(0, \infty)$ and uniformly convergent in $(\epsilon, \infty), \epsilon$ being an arbitrarily small but fixed positive number.

Using Hardy's formula

$$
\sum_{n=0}^{\infty} \lambda_{n}^{-1} L_{n}^{(\alpha)}(x) L_{n}^{(\alpha)}(y) \theta^{n}=(1-\theta)^{-1}(\theta x y)^{-\frac{\alpha}{2}} \exp \left[-\frac{\theta(x+y)}{1-\theta}\right] I_{\alpha}\left[\frac{2(\theta x y)^{1 / 2}}{1-\theta}\right],
$$

where $I_{\alpha}$ denotes the modified Bessel function of the first kind, as the generating function for the series solution (13), we get

$$
f(x, s ; y, t)=\theta^{-\frac{\alpha}{2}}(1-\theta)^{-1} \exp \left[-\frac{(1+\theta)(x+y)}{2(1-\theta)}\right] I_{\alpha}\left[\frac{2(\theta x y)^{1 / 2}}{1-\theta}\right] .
$$

The modified Bessel function $I_{\alpha}(z)$ is defined by

$$
I_{\alpha}(z)=i^{-\alpha} J_{\alpha}(i z)=\sum_{n=0}^{\infty} \frac{\left(\frac{1}{2} z\right)^{\alpha+2 n}}{n !(\alpha+n) !}
$$

where $z$ is positive and $\alpha$ is an integer.

For $\alpha$ a positive integer or zero, a condition which is not incompatible with the assumption $\alpha>-1, I_{\alpha}(z)$ is positive. Thus, according to (14), the transition probability $f$ is always positive for any values of $x$ and $y$ in the interval $(0, \infty)$. Therefore, the solution (13) satisfies the condition (5) provided $\alpha$ takes positive integral or zero values only.

It has been shown [8,9] that, as a consequence of Kogbetliantz's in equalities [16], the solution 
(13) satisfies the special probabilistic condition

$$
\lim _{t \rightarrow s} f(x, s ; y, t)=0,
$$

for any finite and positive values of $x$ and $y$, and also the condition

$$
\lim _{y \rightarrow x, t \rightarrow s} f(x, s ; y, t)=\infty \text {. }
$$

\section{Limiting Behavior of the Probability}

Let us investigate the asymptotic behavior of the probability $\omega(x, s ; V, t)$, defined by (3), when the time $t$ tends to infinity. To do so the existence of the limit of the transition probability $f$ defined by the uniformly convergent series

$$
f(x, s ; y, t)=\exp \left[-\frac{x+y}{2}\right](x y)^{\frac{\alpha}{2}}\left\{\Gamma^{-1}(\alpha+1)+\sum_{n=1}^{\infty} \lambda_{n}^{-1}\left[\frac{a(s)}{a(t)}\right]^{n} L_{n}^{(\alpha)}(x) L_{n}^{(\alpha)}(y)\right\}
$$

should be sought. Having in mind that in the factor $\theta(s, t)=a(s) / a(t)$ the function $a(t)$ is a positive increasing continuous function of $t$, two cases may occur:

1.

$$
\lim _{t \rightarrow \infty} a(t)=\infty
$$

hence

$$
\lim _{t \rightarrow \infty} \theta(s, t)=0 .
$$

Therefore, for $x, y$, and $s$ fixed and $t$ tending to infinity we have

$$
\lim _{t \rightarrow \infty} f(x, s ; y, t)=\Gamma^{-1}(\alpha+1)(x y)^{\frac{\alpha}{2}} \exp [-(x+y) / 2]
$$

which depends on $x$ and $y$. It follows from (3) that the limit of the probability $\omega(x, s ; V, t)$, where $V$ is the interval $(0, \infty)$, is given by

$$
\lim _{t \rightarrow \infty} \omega(x, s ; V, t)=2^{\frac{\alpha}{2}+1} \Gamma\left(\frac{\alpha}{2}+1\right) \Gamma^{-1}(\alpha+1) x^{\frac{\alpha}{2}} \exp \left[-\frac{x}{2}\right]
$$

provided $\alpha>-2$. This limit depends on the initial state $x$ which is called a nonoscillatory case. This terminology is due to M. Fréchet, and it means that the probability $\omega$ tends to a limit, in the ordinary sense, when $t$ tends to infinity.

By assumption, $\alpha$ takes positive integers or zero values, and therefore the condition $\alpha>-2$ is fulfilled.

2. The only alternative to the case 1 is that

$$
\lim _{t \rightarrow \infty} a(t)=d
$$

where $d$ is finite and positive. In this case

$$
\lim _{t \rightarrow \infty} \theta(s, t)=a(s) / d=a_{1}(s)
$$

where $0<a_{1}(s)<1$.

It follows that

$$
\lim _{t \rightarrow \infty} f(x, s ; y, t)=(x y)^{\frac{\alpha}{2}} \exp \left[-\frac{x+y}{2}\right] \sum_{n=0}^{\infty} \lambda_{n}^{-1}\left[a_{1}(s)\right]^{n} L_{n}^{(\alpha)}(x) L_{n}^{(\alpha)}(y),
$$


hence

where

$$
\begin{aligned}
\lim _{t \rightarrow \infty} \omega(x, s ; V, t) & =\int_{V}(x y)^{\frac{\alpha}{2}} \exp \left[-\frac{x+y}{2}\right] \sum_{n=0}^{\infty} \lambda_{n}^{-1}\left[a_{1}(s)\right]^{n} L_{n}^{(\alpha)}(x) L_{n}^{(\alpha)}(y) d y \\
& =\sum_{n=0}^{\infty} K_{n}\left[a_{1}(s)\right]^{n} \psi_{n}^{(\alpha)}(x)
\end{aligned}
$$

$$
K_{n}=\int_{0}^{\infty} \psi_{n}^{(\alpha)}(y) d y
$$

Therefore the limit of the probability $\omega$ depends completely on the initial state $x$ at the instant $s$. This second case is also a nonoscillatory case.

The above results can be stated as follows:

THEOREM. If the transition probability $f(x, s ; y, t)$ satisfying the Chapman-Kolmogoroff eq (12), is in the form (13), then the limit of the probability $\omega$, when $t$ tends to infinity, exists and depends on the initial state, i.e., the probability is nonoscillatory, provided $\alpha$ takes zero or positive integral values.

\section{Stochastic Partial Differential Equations}

A. Einstein [3] showed that the probability density $f$ satisfies a partial differential equation of diffusion type whose coefficient is a certain diffusion constant related to the viscosity and temperature of the medium in which Brownian motion takes place, and to the radius of the Brownian particle (assumed spherical).

The existence of the probability density $f$ has been established by many authors from different viewpoints, especially by A. N. Kolmogoroff [17] and W. Feller [4]. Kolmogoroff proved that any function which satisfies the Chapman-Kolmogoroff equation satisfies also a second-order partial differential equation of parabolic type, and he obtained a class of particular solutions and showed that these, subject to certain special conditions concerning the order of the magnitude of moments, satisfy, in variables $x$ and $s$, the equation

$$
B^{2}(x, s) \frac{\partial^{2} f}{\partial x^{2}}+A(x, s) \frac{\partial f}{\partial x}+\frac{\partial f}{\partial s}=0
$$

where

$$
\begin{aligned}
& B^{2}(x, t)=\lim _{\Delta t \rightarrow 0} \frac{1}{2 \Delta t} \int_{-\infty}^{+\infty}(y-x)^{2} f(x, t, t+\Delta t, y) d y, \\
& A(x, t)=\lim _{\Delta t \rightarrow 0} \frac{1}{\Delta t} \int_{-\infty}^{+\infty}(y-x) f(x, t, t+\Delta t, y) d y,
\end{aligned}
$$

under the assumption that the ratio of the moment of order 3 to that of order 2 tends to zero at the limit $\Delta t=0$.

Kolmogoroff's partial differential equation was derived by C. M. Tchen [20] independently of Kolmogoroff's method.

It can be shown that the solution (13) is different from those discussed by Kolmogoroff.

To see this, note that if the solution (13) belongs to those of Kolmogoroff, then there would exist two functions $A$ and $B$ such that the solution (13) satisfies a partial differential equation of type (15). Calculating the derivatives of $f$, given by (13), we get

$$
\begin{aligned}
& \frac{\partial f}{\partial s}=(x y)^{\frac{\alpha}{2}} \exp \left[-\frac{x+y}{2}\right] \sum_{n \geq 0} n \frac{\theta^{n-1}}{\lambda_{n}} \frac{\partial \theta}{\partial s} L_{n}^{(\alpha)}(x) L_{n}^{(\alpha)}(y) \\
& \frac{\partial f}{\partial x}=(x y)^{\frac{\alpha}{2}} \exp \left[-\frac{x+y}{2}\right] \sum_{n \geq 0} \frac{\theta^{n}}{\lambda_{n}}\left[\left(\frac{\alpha}{2 x}-\frac{x}{2}\right) L_{n}^{(\alpha)}(x)+L_{n}^{\prime(\alpha)}(x)\right] L_{n}^{(\alpha)}(y) \\
& \frac{\partial^{2} f}{\partial x^{2}}=(x y)^{\frac{\alpha}{2}} \exp \left[-\frac{x+y}{2}\right] \sum_{n \geq 0} \frac{\theta^{n}}{\lambda_{n}}\left[L_{n}^{\prime \prime(\alpha)}(x)+\left(\frac{\alpha}{x}-x\right) L_{n}^{\prime(\alpha)}(x)+\left(\frac{x^{2}}{4}+\frac{\alpha^{2}-2 \alpha}{4 x^{2}}-\frac{\alpha+1}{2}\right) L_{n}^{(\alpha)}(x)\right] L_{n}^{(\alpha)}(y),
\end{aligned}
$$

where prime denotes the derivative with respect to $x$. 
For $x$ and $y$ varying in $(0, \infty), t$ fixed, and $s$ lying in a bounded interval such that $s<t$, the series, obtained by differentiating (13) term by term, are uniformly convergent. Substituting the above derivatives in (15), one gets

$$
\exp \left[-\frac{x+y}{2}\right](x y)^{\frac{\alpha}{2}} \sum_{n=0}^{\infty} \frac{\theta^{n}}{\lambda_{n}}\left[U_{n}(x, s, t)\right] L_{n}^{(\alpha)}(y)=0
$$

where $U_{n}(x, s, t)$ denotes

$$
\begin{aligned}
U_{n}(x, s, t)=\frac{n}{\theta} \frac{\partial \theta}{\partial s} L_{n}^{(\alpha)}(x)+A(x, s) & {\left[L_{n}^{\prime(\alpha)}(x)+\left(\frac{\alpha}{2 x}-\frac{x}{2}\right) L_{n}^{(\alpha)}(x)\right] } \\
+ & B^{2}(x, s)\left[L_{n}^{\prime \prime(\alpha)}(x)+\left(\frac{\alpha}{x}-x\right) L_{n}^{\prime(\alpha)}(x)+\left(\frac{x^{2}}{4}+\frac{\alpha^{2}-2 \alpha}{4 x^{2}}-\frac{\alpha+1}{2}\right) L_{n}^{(\alpha)}(x)\right] .
\end{aligned}
$$

Since the relation (16) must hold for any value of $y$ in $(0, \infty)$, the expression in the square brackets must be zero for any positive integer value of $n$; that is, after dividing by $L_{n}^{(\alpha)}(x)$,

$$
\frac{n}{\theta} \frac{\partial \theta}{\partial s}+A\left[\frac{L_{n}^{\prime(\alpha)}(x)}{L_{n}^{(\alpha)}(x)}+\left(\frac{\alpha}{2 x}-\frac{x}{2}\right)\right]+B^{2}\left[\frac{L_{n}^{\prime \prime(\alpha)}(x)}{L_{n}^{(\alpha)}(x)}+\left(\frac{\alpha}{x}-x\right) \frac{L_{n}^{\prime(\alpha)}(x)}{L_{n}^{(\alpha)}(x)}+\left(\frac{x^{2}}{4}+\frac{\alpha^{2}-2 \alpha}{4 x^{2}}-\frac{\alpha+1}{2}\right)\right]=0 .
$$

As $\frac{1}{\theta} \frac{\partial \theta}{\partial s}$ is independent of $n$ and $x$, it is clear that the relation (17) does not hold unless the two coefficients $A(x, s)$ and $B^{2}(x, s)$ are both zero. Therefore $\theta(s, t)=a(s) / a(t)$ will be independent of $s$ which is contrary to the assumption that $a(s)$ is a positive increasing continuous function of $s$. This proves the statement.

It can also be shown by computation that the relation (17) is possible if the two functions $A(x, s)$ and $B(x, s)$ are both zero. In fact for $n=0$, we have

$$
\frac{A}{B^{2}}=\frac{x^{4}+\alpha^{2}-2 \alpha-2 x^{2}(\alpha+1)}{2 x\left(x^{2}-\alpha\right)} .
$$

On the other hand, setting $n=1$ and $n=2$ in relation (17) and eliminating $\frac{1}{\theta} \frac{\partial \theta}{\partial s}$ between the two relations obtained, one gets

where

$$
\frac{A}{B^{2}}=\frac{C}{D}
$$

and

$$
C=\frac{2\left(\alpha-x^{2}\right)}{x(x-\alpha-1)}+\frac{x^{4}+\alpha^{2}-2 \alpha}{4 x^{2}}-\frac{\alpha+1}{2}-\frac{2 x+2\left(\alpha-x^{2}\right)(x-\alpha-2)}{x^{3}-2(\alpha+2) x^{2}+(\alpha+2)(\alpha+1) x}
$$

$$
D=\frac{2(x-\alpha-2)}{x^{2}-2(\alpha+2) x+(\alpha+2)(\alpha+1)}-\frac{2}{(x-\alpha-1)}+\frac{x}{2} .
$$

The two ratios (18) and (19) are different, hence the relation (17) does not hold unless the two functions $A$ and $B$ are both zero.

Now we propose to obtain a derivation of the corresponding parabolic partial differential equation or the so-called backward equation of diffusion theory. For this purpose we can use the generating function (14) and get

where

$$
I_{\alpha}(z)=\theta^{\frac{\alpha}{2}}(1-\theta) \exp \left[\frac{(1+\theta)(x+y)}{2(1-\theta)}\right] f(x, s ; y, t)
$$

and $I_{\alpha}(z)$ satisfies

$$
z=2(1-\theta)^{-1}(\theta x y)^{1 / 2},
$$

$$
z^{2} I_{\alpha}^{\prime \prime}(z)+z I_{\alpha}^{\prime}(z)-\left(\alpha^{2}+z^{2}\right) I_{\alpha}(z)=0
$$

prime denotes the derivative with respect to $z$. 
Taking the derivatives of (20) with respect to $x$ and computing the different terms of (21), one gets

$$
\begin{aligned}
z I_{\alpha}^{\prime} & =2 x \theta^{\frac{\alpha}{2}}(1-\theta) \exp \left[\frac{(1+\theta)(x+y)}{2(1-\theta)}\right]\left[\frac{\partial f}{\partial x}+\frac{1+\theta}{2(1-\theta)} f\right], \\
z^{2} I_{\alpha}^{\prime \prime} & =4 x^{2} \theta^{\frac{\alpha}{2}}(1-\theta) \exp \left[\frac{(1+\theta)(x+y)}{2(1-\theta)}\right]\left\{\frac{\partial^{2} f}{\partial x^{2}}+\frac{\partial f}{\partial x}\left(\frac{1+\theta}{1-\theta}+\frac{1}{2 x}\right)+f\left[\frac{(1+\theta)^{2}}{4(1-\theta)^{2}}+\frac{1+\theta}{4 x(1-\theta)}\right]\right\}, \\
\left(\alpha^{2}+z^{2}\right) I_{\alpha} & =\theta^{\frac{\alpha}{2}}(1-\theta) \exp \left[\frac{(1+\theta)(x+y)}{2(1-\theta)}\right] \frac{\alpha^{2}(1-\theta)^{2}+4 \theta x y}{(1-\theta)^{2}} f .
\end{aligned}
$$

Substituting in (21), and dividing by $\theta^{\frac{\alpha}{2}}(1-\theta) \exp \left[\frac{(1+\theta)(x+y)}{2(1-\theta)}\right]$ we have

$$
4 x^{2} \frac{\partial^{2} f}{\partial x^{2}}+4 x\left[\frac{x(1+\theta)}{1-\theta}+1\right] \frac{\partial f}{\partial x}+\frac{x^{2}(1+\theta)^{2}+2 x\left(1-\theta^{2}\right)-\alpha^{2}(1-\theta)^{2}-4 \theta x y}{(1-\theta)^{2}} f=0 .
$$

The relations

$$
\frac{\partial I_{\alpha}}{\partial x}=I_{\alpha}^{\prime} \frac{\partial z}{\partial x} \text { and } \frac{\partial I_{\kappa}}{\partial \theta}=I_{\alpha}^{\prime} \frac{\partial z}{\partial \theta}
$$

lead us to express $f$ in terms of $\frac{\partial f}{\partial x}$ and $\frac{\partial f}{\partial \theta}$ as follows:

$$
f=\frac{2(1-\theta)}{(1+\theta) x-\alpha(1-\theta)^{2}+2 \theta(1-\theta)-2 \theta(x+y)}\left(\theta \frac{\partial f}{\partial \theta}-x \frac{\partial f}{\partial x}\right) .
$$

Substituting the above expression of $f$ in (22) and setting $\beta(x, s)=2 x^{2}(1-\theta)\left[(1+\theta) x-\alpha(1-\theta)^{2}+2 \theta(1-\theta)-2 \theta(x+y)\right]\left[x^{2}(1+\theta)^{2}\right.$

and

$$
\left.+2 x\left(1-\theta^{2}\right)-\alpha^{2}(1-\theta)^{2}-4 \theta x y\right]^{-1}
$$

$\gamma(x, s)=x\left\{2[x(1+\theta)+(1-\theta)]\left[(1+\theta) x-\alpha(1-\theta)^{2}+2 \theta(1-\theta)-2 \theta(x+y)\right]\left[x^{2}(1+\theta)^{2}\right.\right.$

one gets

$$
\left.\left.+2 x\left(1-\theta^{2}\right)-\alpha^{2}(1-\theta)^{2}-4 \theta x y\right]^{-1}-1\right\},
$$

On the other hand

$$
\beta(x, s) \frac{\partial^{2} f}{\partial x^{2}}+\gamma(x, s) \frac{\partial f}{\partial x}+\theta \frac{\partial f}{\partial \theta}=0 .
$$

so that

$$
\frac{\partial f}{\partial s}=\frac{\partial f}{\partial \theta} \frac{\partial \theta}{\partial s}=\frac{a^{\prime}(s)}{a(t)} \frac{\partial f}{\partial \theta}
$$

$$
\theta \frac{\partial f}{\partial \theta}=\frac{a(s)}{a(t)} \frac{a(t)}{a^{\prime}(s)} \frac{\partial f}{\partial s}=\frac{a(s)}{a^{\prime}(s)} \frac{\partial f}{\partial s}
$$

where prime denotes the derivative with respect to $s$. Hence equation (23) becomes

$$
\beta \frac{\partial^{2} f}{\partial x^{2}}+\gamma \frac{\partial f}{\partial x}+\frac{a(s)}{a^{\prime}(s)} \frac{\partial f}{\partial s}=0 .
$$

This derivation presupposes certain differentiability conditions on $f$ and $a(s)$, namely: $f$ must have continuous partial derivatives of order $\leq 2$ with respect to $x$ and $s$, and the righthanded derivative $a^{\prime}(s)$ must exist. Therefore the following theorem can be stated:

Theorem. If the transition probability function $f$, defined by $(13)$, is of the class $C^{2}$, and the right-handed derivative $a^{\prime}(s)$ of the increasing continuous function a $(s)$ exists, and is everywhere positive and different from zero, then the transition probability $f$ satisfies, in the variables $x$ and $s$, the parabolic equation (24). 
It can be observed that the parabolic eq (24) to which the solution (13) satisfies is different from equation (15) given by Kolmogoroff.

The coefficient of $\frac{\partial f}{\partial s}$ in (24) suggests setting $a(s)=e^{s}$, and then putting $a(t)=e^{t}$, we have

$$
\theta=e^{s-t} \text {. }
$$

The special case $a(s)=e^{s}$ leads us to a particular solution of eq (12) in the form

$$
\begin{aligned}
f(x, s ; y, t) & =\exp [-(x+y) / 2](x y)^{\frac{\alpha}{2}} \sum_{n=0}^{\infty} \lambda_{n}^{-1} \exp [n(s-t)] L_{n}^{(\alpha)}(x) L_{n}^{(\alpha)}(y) \\
& =\theta^{-\frac{\alpha}{2}}(1-\theta)^{-1} \exp \left[-\frac{(1+\theta)(x+y)}{2(1-\theta)}\right] I_{\alpha}\left[\frac{2(\theta x y)^{\frac{1}{2}}}{1-\theta}\right] .
\end{aligned}
$$

and the eq (24) will be reduced to the normal form

$$
\beta \frac{\partial^{2} f}{\partial x^{2}}+\gamma \frac{\partial f}{\partial x}+\frac{\partial f}{\partial s}=0
$$

If we now set, more generally,

$$
a(s)=e^{S}
$$

where the variable $S$ increases with $s$, then

and

$$
\begin{aligned}
& a(t)=e^{T} \\
& s=\mu(S) \\
& t=\mu(T)
\end{aligned}
$$

where $\mu(S)$ is an increasing continuous function of $S$. We then have

$$
\theta=e^{S-T} .
$$

The function $f(x, s ; y, t)$ becomes $F(x, S ; y, T)$, and the Chapman-Kolmogoroff equation will still be satisfied if the instants $s, t$ are replaced by numbers $S$ and $T$ and the function $F(x, S ; y, T)$ can be written as follows:

$$
F(x, S ; y, T)=\int_{0}^{\infty} F(x, S ; z, U) F(z, U ; y, T) d z, \quad S<U<T .
$$

The series solution (13) now becomes

$$
\begin{aligned}
f(x, s ; y, t) & =f[x, \mu(S) ; y, \mu(T)]=F(x, S ; y, T) \\
& =\exp \left[-\frac{x+y}{2}\right](x y)^{\frac{\alpha}{2}} \sum_{n=0}^{\infty} \lambda_{n}^{-1} \exp [n(S-T)] L_{n}^{(\alpha)}(x) L_{n}^{(\alpha)}(y) .
\end{aligned}
$$

When the time $t \rightarrow \infty, T$ tends either to infinity or ( $T$ being negative as well as $S$ ) to zero, then $\theta$ tends either to zero or $e^{S}$, so that the limiting behavior of the probability function $\omega$, defined by

will still be nonoscillatory.

$$
\omega=\int_{0}^{\infty} F(x, S ; y, T) d y
$$

There is nothing arbitrary in $F(x, S ; y, T)$, defined by (28), which is a particular solution of (27). Therefore the most general solution will be in the form

$$
f(x, s ; y, t)=F[x, \eta(s) ; y, \eta(t)]
$$

where $\eta(s)=\ln a(s)$ is an arbitrary function varying in $(-\infty, \infty)$. 


\section{References}

[1] S. Chapman, Proc. Roy. Soc. A119, 34-54 (1928).

[2] J. L. Doob, Trans. Am. Math. Soc. 42, 107-140 (1937).

[3] A. Einstein, Ann. Phys. 17, 549 (1905).

[4] W. Feller, Math. Ann. 113, 113-160 (1936).

[5] W. Feller, Trans. Am. Math. Soc. 7\%, 1-31 (1954).

[6] M. Fréchet, Proc. London Math. Soc. 39, 515-540 (1935).

[7] M. Fréchet, Compt. Rend. Acad. Sci. Paris 200, 369 (1935).

[8] A. Ghaffari, Doctoral Thesis, Math., Paris Univ. (1936), Gauthier-Villars, Paris.

[9] A. Ghaffari, Bull. Am. Math. Soc. 58, 43 (1952).

[10] A. Ghaffari, Proc. of the Inter. Conf. on Partial Differential Equations and Continuum Mechanics, 348 350, held at the University of Wisconsin, Madison, Wis., June 1960. The Univ. of Wisconsin Press, 1961.

[11] B. Hostinsky, Mémorial des Sciences Math., Fasc. 52 (1931).

[12] M. Kac, Am. Math. Monthly 54, 369-391 (1947).

[13] M. Kac, Lectures in Applied Math., Proc. of the Summer Seminar, Boulder, Colo. (1957), Vol. I, Interscience Publishers.

[14] S. Kakutani, Proc. Japan Acad. 21, 138 (1945).

[15] S. Kakutani, Proc. Nat. Acad. Sci. U.S.A. 36, 319 (1950).

[16] E. Kogbetlintz, J. Math. and Phys. 14, 1-99 (1935).

[17] A. N. Kolmogoroff, Math. Ann. 104, 415-458 (1931).

[18] P. Lévy, Am. J. Math. Vol. 62, 487 (1948).

[19] M. Smoluchovski, Phys. Z. 17, 557-571 (1916).

[20] C. M. Tchen, Vers. Ned. Akad. V. Wetensch., Afd. Natuurk. 53, 400-410 (1944).

[21] G. E. Uhlenbeck and L. S. Ornstein, Phys. Rev. 36, 823-841 (1930).

[22] M. C. Wang and G. E. Uhlenbeck, Rev. Modern Phys. 17, 323-342 (1945).

[23] N. Wiener, Publications of M.I.T., series II, No. 60 (1923).

(Paper 65B4-64) 